\title{
Surgical Management of Traumatic Epidural Hematomas
}

\author{
NAEEM UL HAQ ${ }^{1}$, ADNAN AHMED ${ }^{2}$, MUKHTIYAR ALI ${ }^{3}$, MUHAMMAD ISHAQ $^{4}$, SANA ULLAH ${ }^{5}$, WAQAS MEHDI \\ MEHTAABUDIN ${ }^{7}$ \\ ${ }^{1}$ Assistant Professor and Chairman Neurosurgery, Bacha Khan Medical College Mardan Medical Complex \\ ${ }^{2}$ Registrar Neurosurgery Ward, Bacha Khan Medical College Mardan Medical Complex \\ ${ }^{3}$ Professor of Surgery, Bacha Khan Medical College, Mardan Medical Complex \\ ${ }^{4}$ Assistant Professor Neurosurgery, Bacha Khan Medical College Mardan Medical Complex \\ ${ }^{5}$ Resident of Neurosurgery Department, King Edward Medical University/Mayo Hospital Lahore \\ ${ }^{6}$ Assistant Professor, Department of Neurosurgery, King Edward Medical University/Mayo Hospital Lahore \\ ${ }^{7}$ Resident of Neurosurgery Department, King Edward Medical University/Mayo Hospital, Lahore \\ Correspondence to Dr Naeem UI Haq,
}

\begin{abstract}
Aim: To find out predictive significance and impact on neurosurgical treatment in EDH patients

Setting \& duration of study: Neurosurgery unit, Mardan Medical Complex, Mardan, from Dec, 2017 to Dec, 2019

Sample size: 120 individuals with traumatic epidural hematoma were treated through surgical intervention.

Result: Out of 120 cases, $74 \%$ were male, while $26 \%$ were female. Motor vehicle accidents caused $86 \%$ of the injuries, while falls and heavy hits caused $14 \%$ of the injuries. On well defined tomography imaging of the brain, $14 \%$ individuals exhibited the swirl sign. The epidural hematoma had a strong association with preoperative Glasgow score, preoperative mydriasis, time from injury to CT scan, and intraoperative hematoma volume.

Conclusion: Epidural hematoma has an excellent outcome, if managed on time. The epidural hematoma may be linked with a cataclysmic result, with comparatively high rates of mortality and morbidity.

Keywords: Epidural hematoma, Glasgow Outcome Scale
\end{abstract}

\section{INTRODUCTION}

In patients of epidural hematoma, important source of bleeding is the middle meningeal artery ${ }^{1}$. Brain herniation and midbrain injury, as well as mortality owing to respiratory arrest, could result from the speedy hematoma extension and considerably elevated pressure inside the cranium. On CT scans of brain, the swirl sign is recognized as a low attenuation area inside the cranium hyper attenuating liquid retention, which provides a symptom of vigorous bleeding that requires instant surgical intervention. Despite the fact that the radiological imaging of the swirl sign in epidural hematoma has been precisely identified, its medical importance is unknown ${ }^{2,3}$.

The objective of the study was to find out predictive significance and impact on neurosurgical treatment in EDH patients

\section{METHODOLOGY}

This prospective study was conducted in Neurosurgery Unit, Mardan Medical Complex, Mardan from April 2020 and April 2021 after approval from Ethical Review Committee. The study excluded subjects who sustained open or mixed lesion or who had previous neurological defects. Demographic characteristics (age and sex), time period of injury and its mechanism, coma scale of Glasgow score, eyes response, and radiological imaging were collected. All patients were assessed and immediately sent to the OT for surgical management of hematoma inside the cranium. The following criteria were indicated for surgery:

- $\quad$ Displacement of midline $>5 \mathrm{~mm}$

- Thickness of clot $>10 \mathrm{~mm}$

- Hematoma volume $>30 \mathrm{ml}$

Received on 28-04-2021

Accepted on 27-07-2021
- Compressed basal cisterns

- Neurological impairment

Removal of Hematoma was accomplished using a huge skin flap of question mark shaped and a big frontal and temporal craniotomy through conventional craniotomy. Following hematoma excision, a tiny incision was performed in the epidural membrane to figure out the possibility of an essential subdural hematoma. Adverse outcome was characterized statistically as a GOS score of 1,2 , or 3 , and approving measure was defined as a Glasgow score of 4 and 5. In EDH, the swirl sign is defined as a tiny hypo attenuating zone with a swirling structure contained within a big extradural clot. The study was conducted using SPSS version 25.0.

\section{RESULTS}

The detail of results is given in table 1 and Fig 2,3

Fig 1: Pre Operative imaging
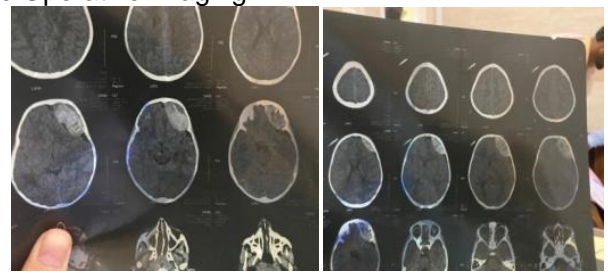

Fig 2: Post Operative imaging
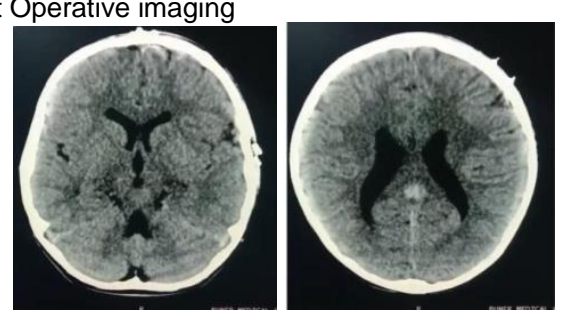
Fig. 3: M:F ratio in epidural hematoma

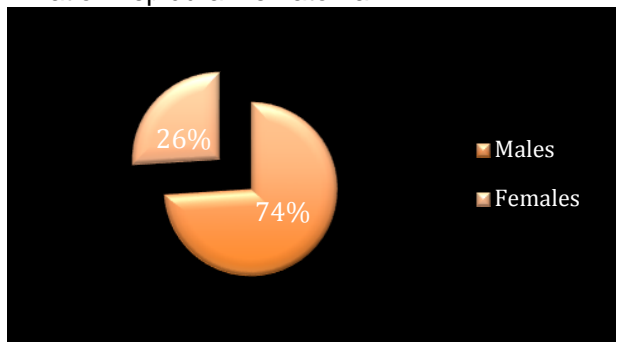

Fig. 4: Factors of epidural hematoma

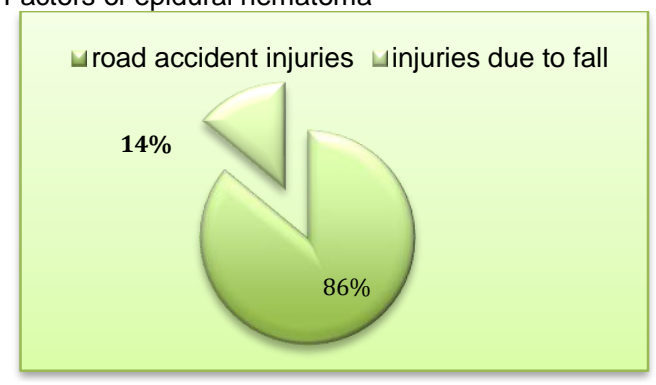

Table 1: Data analysis

\begin{tabular}{|l|l|l|}
\hline Variables & & P value \\
\hline Males & $74 \%$ & 0.041 \\
\hline Females & $26 \%$ & 0.03 \\
\hline Road Accident Injuries & $86 \%$ & 0.043 \\
\hline Fall Injuries & $14 \%$ & 0.06 \\
\hline Glasgow score at admission & $1,2,3$ & 0.001 \\
\hline Preoperative Glasgow score & $1,2,3,4$ & 0.024 \\
\hline Preoperative Mydriasis & $33 \%$ & 0.05 \\
\hline Time Period (Injury to CT scan) & $2-6$ hours & 0.044 \\
\hline Hematoma volume on CT scan & $65.9 \mathrm{ml}$ & 0.03 \\
\hline Hematoma Volume intraoperative & $78.7 \mathrm{ml}$ & 0.185 \\
\hline Intracranial Fracture & $39 \%$ & 0.01 \\
\hline
\end{tabular}

\section{DISCUSSION}

Our findings indicate that the AEDH is related with a higher death rate and a poor neurological outcome 12 weeks after injury. Greenberg et $\mathrm{al}^{5}$ established that the epidural hematoma in extra-axial cerebral hematomas indicated an acute, fast, and continuous hemorrhage and was linked with much greater fatality and morbidity rates. Ono et $\mathrm{al}^{4}$ studied patients with severe head injury retrospectively and concluded that the Glasgow score was the sole meaningful predictive factor in the EDH group.

Lee et $\mathrm{al}^{7}$ studied patients who underwent neurosurgery for AEDH and saw that postoperative consciousness state, Glasgow score, and pupillary size all had a significant link with functional prognosis. Prior to surgery, patients with the epidural hematoma exhibited more severe clinical symptoms, i.e. a lower preoperative Glasgow score and mydriasis, which may have contributed to their poor result. The time interval between the trauma and the CT scan is definitely critical in predicting the incidence of the swirl sign.

In our study, patients who had a CT scan within two hours of injury had a higher prevalence of the swirl sign than patients who had a CT scan $>6$ hours after damage. Thus, the sooner a CT scan is performed following head trauma, the greater the chances of detecting the hematoma on CT. Temporal or temporoparietal EDHs were the most prevalent locations in patients with or without the swirl sign. They were produced by a fracture of the pterion with tearing of the MMA and/or middle meningeal vein. In another study, the actual intraoperative hematoma volume was greater in patients with the swirl sign than in patients without the swirl sign, despite the lack of a significant correlation between the swirl sign and the estimated hematoma volume on CT scan ${ }^{8}$.

Tian et $\mathrm{al}^{9}$ found that the time interval between trauma and surgical decompression was correlated with mortality, the sooner decompression occurred in cases of acute head trauma, the better the chance of a favorable outcome.

We recommend that patients, who require surgery, receive surgical evacuation and hemostasis as quickly as possible after trauma. Adequate exposure via the craniotomy is also necessary for seeing and controlling the bleeding source.

\section{CONCLUSION}

The epidural hematoma on CT imaging throughout the early clinical course may be linked with a cataclysmic result, with comparatively high rates of mortality and morbidity. The epidural hematoma had a strong association with preoperative Glasgow score, preoperative mydriasis, time from injury to CT scan, and intraoperative hematoma volume.

\section{Conflict of interest: Nil}

\section{REFERENCES}

1. Cheung PS, Lam JM, Yeung JH et al. (2007) Outcome of traumatic extra dural hematoma in Hong Kong. Injury38:7680

2. Zimmerman RA, Bilaniuk LT (1982) Computed tomographic staging of traumatic epidural bleeding. Radiology 144:809812

3. Al-Nakshabandi NA (2001) The swirl sign. Radiology 218:433

4. Brain Trauma Foundation, American Association of Neurological Surgeons, Congress of Neurological Surgeons and Joint Section on Neurotrauma and Critical Care (2007) Guidelines for the Neurol Sci (2017) 38:2111-2116

5. Greenberg J, Cohen WA, Cooper PR (1985) The hyper acute extra axial intracranial hematoma: computed tomographic findings and clinical significance. Neurosurgery 17:48-56

6. Ono J, Yamaura A, Kubota M et al. (2001) Outcome prediction in severe head injury: analyses of clinical prognostic factors. J Clin Neuro sci 8:120-123

7. Lee EJ, Hung YC, Wang LC et al. (1998) Factors influencing the functional outcome of patients with acute epidural hematomas: analysis of 200 patients undergoing surgery. $\mathrm{J}$ Trauma45:946-952

8. Bullock MR, Chesnut R, Ghajar J et al (2006) Surgical management of acute epidural hematomas. Neurosurgery 58(3 suppl):S7-15

9. Tian HL, Chen SW, Xu T et al (2008) Risk factors related to hospital mortality in patients with isolated traumatic acute subdural hematoma: analysis of 308 patients undergone surgery. Chin Med J 121:1080-1084. 